\title{
V. Starch and unannealed glass under the polariscope
}

\section{Walter Baily}

To cite this article: Walter Baily (1879) V. Starch and unannealed glass under the polariscope, Philosophical Magazine Series 5, 7:40, 39-50, DOI: 10.1080/14786447908639551

To link to this article: http://dx.doi.org/10.1080/14786447908639551

曲 Published online: 13 May 2009.

Submit your article to this journal ๘

Џll Article views: 2

Q View related articles $\longleftarrow$ 
The phase-displacements $\alpha, \alpha_{1}, \boldsymbol{\alpha}_{0}$ are accordingly small quantities of the same order, which in the limiting case

become $=0$.

$$
\frac{\mathrm{W}}{2 \pi n \mathrm{Q}}=\frac{\mathrm{W}_{1}}{2 \pi n \mathrm{Q}_{1}}=\frac{\mathrm{W}_{0}}{2 \pi n \mathrm{Q}_{0}}=0
$$

But the results deduced for this special case are the expression of those obtained experimentally by M. Hermann. If it can be shown that in fact the quantities $\frac{\mathrm{W}}{2 \pi n \mathrm{Q}}$ and $\frac{\mathrm{W}_{1}}{2 \pi n \mathrm{Q}_{1}}$ were very small values in his experiments, then the complete agreement of the results of experiment with the general law of induction is demonstrated. By a closer consideration of the dimensions, the number of turns, and the resistances of the coils and telephones employed by him, we can perceive that the quotients mentioned actually were small values.

\section{Starch and Unannealed Glass under the Polariscope.} By Walter Bally*.

\section{[Plates I.-IV.]}

THERE are many bodies, of which a spherical grain of 1 starch and a circular plate of unannealed glass may be taken as specimens, having an optical structure symmetrical about an axis through the body. The object of this paper is to investigate the state of the light which emerges from such a body, when monochromatic light in any state of polarization is sent through the body in the direction of the axis.

In fig. 1 (Plate I.) let $\mathrm{S} \mathrm{S}^{\prime}$ and $\mathrm{T} \mathrm{T}^{\prime}$ be drawn through $\mathrm{R}$ perpendicular to one another, and let $U^{U^{\prime}}$ and $V V^{\prime}$ bisect the angles between them. Suppose a quarter-undulation plate to be fixed parallel to the paper, with its axes parallel to $\mathrm{S} \mathrm{S}^{\prime}$ and $\mathrm{T}^{\prime} \mathrm{T}^{\prime}$ - and the light to be passed perpendicularly to the paper through a Nicol's prism having its axis perpendicular to the paper and its plane of polarization inclined at an angle $\rho$ to the line $\mathbf{S} \mathbf{S}^{\prime}$, then through the quarter-undulation plate, and then through the body, which is also to be placed with its axis perpendicular to the paper.

Let the paper represent a section of the light after it has emerged from the body. Take any point $P$ and draw round it an ellipse representing the polarization of the light at $P$. The state of the light will be completely determined if we know the angle $(\alpha)$ which the axis major of this ellipse makes with $\mathrm{RP}$, the angle $(\beta)$ which a line joining the extremities

* Read before the Physical Society, June 20, 1878. 
of the axes of the ellipse makes with the axis major, and the direction in which the rotation takes place.

Let the angle $\mathrm{S} R \mathrm{P}=\phi$; produce $\mathrm{R} \mathrm{P}$ to $\mathrm{X}$ and draw $\mathrm{P} \mathrm{Y}$ $\perp \mathrm{PX}$; take any point $\mathrm{Q}$ on the ellipse, let $\mathrm{P} Q=r$, and the angle $\mathrm{Q} P \mathrm{X}=\theta$.

The resolved part of the vibration at $P$ along $R P$ has been retarded in passing through the body differently to the resolved part perpendicular to $R P$. Let the resolved part along $R P$ have been retarded by a quantity $\sigma$ more than the mean amount, and the other part have been retarded by the same quantity less than the mean amount. The quantity $\sigma$ is a function of the distance $R P$.

Let $\sin t$ represent the vibration in the xther after the light has passed through the Nicol. This is equivalent to

and $\cos \rho \sin t \quad \| \mathrm{SS}^{\prime}$

$$
\sin \rho \sin t \quad \| \mathrm{TT}^{\prime} \text {. }
$$

After passing through the quarter-undulation plate the vibrations become

and

$$
\cos \rho \sin \left(t+45^{\circ}\right) \quad \| S^{\prime}
$$

$$
\sin \rho \sin \left(t-45^{\circ}\right) \quad \| \mathrm{TT}^{\prime} .
$$

These may be written (the coefficient $\frac{1}{\sqrt{ } 2}$ being omitted)

and

$$
\cos \rho(\sin t+\cos t) \quad \| \mathrm{SS}^{\prime}
$$

$$
\sin \rho(\sin t-\cos t) \quad \| \mathrm{TT}^{\prime} .
$$

It is easy to show that these vibrations represent motion in an ellipse whose axes are parallel to $\mathrm{SS}^{\prime}$ and $\mathrm{TT}$, and the extremities of whose axes are joined by a line making an angle $\rho$ with the axis major.

These vibrations are equivalent to

and

$$
\cos (\rho-\phi) \sin t+\cos (\rho+\phi) \cos t \quad \| \mathrm{PX}
$$

$$
\sin (\rho-\phi) \sin t-\sin (\rho+\phi) \cos t \quad \| \mathrm{PY} .
$$

After passing through the body the displacement $\| \mathrm{PX}$ is $r \cos \theta$, and that $\| \mathrm{PY}$ is $r \sin \theta$. Hence

$$
\begin{aligned}
& r \cos \theta=\cos (\rho-\phi) \sin (t+\sigma)+\cos (\rho+\phi) \cos (t+\sigma), \\
& r \sin \theta=\sin (\rho-\phi) \sin (t-\sigma)-\sin (\rho+\phi) \cos (t-\sigma) ;
\end{aligned}
$$

which may be written

$$
\begin{array}{llllll}
r \cos \theta=a \sin t+b \cos t, & . & . & . & . & . \\
r \sin \theta=a^{\prime} \sin t+b^{\prime} \cos t, & . & . & . & . & .
\end{array}
$$


where

$$
\begin{aligned}
& a=\cos (\rho-\phi) \cos \sigma-\cos (\rho+\phi) \sin \sigma, \\
& b=\cos (\rho-\phi) \sin \sigma+\cos (\rho+\phi) \cos \sigma, \\
& a^{\prime}=\sin (\rho-\phi) \cos \sigma-\sin (\rho+\phi) \sin \sigma, \\
& b^{\prime}=-\sin (\rho-\phi) \sin \sigma-\sin (\rho+\phi) \cos \sigma .
\end{aligned}
$$

Differentiating (1) and (2) with respect to $t$, we have

$$
\begin{aligned}
& \cos \theta \frac{d r}{d t}-\sin \theta r \frac{d \theta}{d t}=a \cos t-b \sin t, . . \\
& \sin \theta \frac{d r}{d t}+\cos \theta r \frac{d \theta}{d t}=a^{\prime} \cos t-b^{\prime} \sin t .
\end{aligned}
$$

Maltiplying (3) by $r \sin \theta$, and (4) by $r \cos \theta$, and subtracting, we have

whence

$$
\begin{aligned}
r^{2} \frac{d \theta}{d t} & =(a \sin t+b \cos t)\left(a^{\prime} \cos t-b^{\prime} \sin t\right) \\
& -\left(a^{\prime} \sin t+b^{\prime} \cos t\right)(a \cos t-b \sin t) ;
\end{aligned}
$$

$$
\begin{aligned}
r^{2} \frac{d \theta}{d t} & =a^{\prime} b-a b^{\prime} \\
& =\sin 2 \rho \cos 2 \sigma-\cos 2 \rho \sin 2 \sigma \sin 2 \phi .
\end{aligned}
$$

Again, in (1), (2), (3), (4), let $t$ have a value which makes $r$ a maximum or minimum; then $\theta$ becomes $\alpha$, and $\frac{d r}{d t}$ vanishes.

Eliminating $r$ from (1) and (2), we get

$$
\left(a \sin \alpha-a^{\prime} \cos \alpha\right) \sin t=-\left(b \sin \alpha-b^{\prime} \cos \alpha\right) \cos t ;
$$

and eliminating $r \frac{d \theta}{d t}$ from (3) and (4), we get

Hence

$$
\left(b \cos \alpha+b^{\prime} \sin \alpha\right) \sin t=\left(a \cos \alpha+a^{\prime} \sin \alpha\right) \cos t \text {. }
$$

$$
\frac{a \sin \alpha-a^{\prime} \cos \alpha}{b \cos \alpha+b^{\prime} \sin \alpha}+\frac{b \sin \alpha-b^{\prime} \cos \alpha}{a \cos \alpha+a^{\prime} \sin \alpha}=0,
$$

or

that is,

$$
2\left(a a^{\prime}+b b^{\prime}\right)-\left(a^{2}+b^{2}-a^{2}-b^{\prime 2}\right) \tan 2 \alpha=0 ;
$$

$\sin 2 \rho \sin 2 \sigma+\cos 2 \rho \cos 2 \sigma \sin 2 \phi+\cos 2 \rho \cos 2 \phi \tan 2 \alpha=0$. (6) Now $r^{2} \frac{d \theta}{d t}$ is proportional to the area of the ellipse, as the period of vibration is constant; and the axes of the ellipse are proportional to $\sin \beta$ and $\cos \beta$, since the intensity of the light, and consequently the sum of the squares of the axes is constant over the whole section Hence the area of the ellipse is also proportional to $\sin \beta \cdot \cos \beta$ (that is, to $\sin 2 \beta$ ). Putting 
$\rho=45^{\circ}$ and $\sigma=0$ in (5), we get $r^{2} \frac{d \theta}{d t}=1$. But in this case the emerging light is circularly polarized, and consequently $\beta=45^{\circ}$, whence $\sin 2 \beta$ also equals unity. Hence in every case

Hence

$$
r^{2} \frac{d \theta}{d t}=\sin 2 \beta \text {. }
$$

$\sin 2 \rho \cos 2 \sigma-\cos 2 \rho \sin 2 \sigma \sin 2 \phi-\sin 2 \beta=0 . \quad$.

The locus of points at which the major axis of the ellipse of polarization is inclined at a constant angle to the radius $R P$ will be called an "isoclinal line," and will be denoted by the symbol $K(\alpha)$, where $\alpha$ is this angle. $K(\alpha)$ and $K\left(\alpha-90^{\circ}\right)$ are both included in the equation (6), as that equation does not distinguish between major and minor axes.

The locus of points at which the line joining the extremities of the axes makes a constant angle with the major axis will be called an "isomorphal line," and will be denoted by the symbol $M(\beta)$, where $\beta$ is this angle. Equation (7) is the equation to $\mathrm{M}(\beta)$.

The direction of rotation of the æther is positive or negative according as $\sin 2 \beta$ is positive or negative-that is, according as $\beta$ is positive or negative, since we need only give $\beta$ values lying between $\pm 45^{\circ}$.

In figs. $2,3,4,5$ these loci are represented-the isomorphals by continuous lines, and the isoclinals by dotted lines. The Arabic numerals indicate the values of $\alpha$ in degrees, and the Roman numerals those of $\beta$ in degrees.

The value given to $\sigma$ is $\mathrm{RP}+90^{\circ}$, a function which has been assumed solely for convenience in drawing the figures. The figures are drawn between the limits $\sigma=180^{\circ}$ and $\sigma=360^{\circ}$. An extension beyond these limits would give merely a repetition of the portion within them, since an addition of $180^{\circ}$ to the value of $\sigma$ makes no difference in the equations to the loci. Directions from the centres of these figures will be referred to by means of the lines in fig. 1 .

The values of $\rho$ are in fig. $2,45^{\circ}$, in fig. $3,30^{\circ}$, in fig. 4 , $15^{\circ}$, and in fig. 5 , zero. Accordingly the incident light is circularly polarized in fig. 2, elliptically in figs. 3 and 4 (the eccentricity of the ellipse being less in fig. 3 than in fig. 4), and plane-polarized in fig. 5 .

The isomorphals and isoclinals drawn are those for which the values of $\alpha$ and $\beta$ are $45^{\circ}, 30^{\circ}, 15^{\circ}$, and zero. The thick continuous lines are branches of $M(0)$, which is the locus of plane-polarized light. 'The round spots are loci of circularly polarized light, or $\mathrm{M}( \pm 45)$. 
The symbol $\mathrm{K}( \pm \alpha)$ will be used to include $\mathrm{K}(\alpha), \mathrm{K}\left(\alpha-90^{\circ}\right)$, $K(-\alpha)$, and $K\left(90^{\circ}-\alpha\right)$; its equation is $(\sin 2 \rho \sin 2 \sigma+\cos 2 \rho \cos 2 \sigma \sin 2 \phi)^{2}=\cos ^{2} 2 \rho \cos ^{2} 2 \phi \tan ^{2} 2 \alpha$. (8) And the symbol $\mathrm{M}( \pm \beta)$ will be used to include $\mathrm{M}(\beta)$ and $\mathbf{M}(-\beta)$; its equation is

$(\sin 2 \rho \cos 2 \sigma-\cos 2 \rho \sin 2 \sigma \sin 2 \phi)^{2}=\sin ^{2} 2 \beta$. .

Putting $90^{\circ}-\phi$, or $-90^{\circ}-\phi$, for $\phi$ in these equations makes no change in the equations. Hence $\mathrm{K}( \pm \alpha)$ and $M( \pm \beta)$ are each symmetrical with respect to $U^{U^{\prime}}$ and $\nabla V^{\prime}$.

Adding (8) and (9), we get

$$
\cos ^{2} 2 \phi \cdot \cos ^{2} 2 \rho=\cos ^{2} 2 \alpha \cdot \cos ^{2} 2 \beta
$$

from which equation it appears that the intersections of $K( \pm \alpha)$ with $M( \pm \beta)$ all lie on the two diameters defined by the equation; and since $\alpha$ and $\beta$ are interchangeable in the equation, we see that $K( \pm \gamma)$ and $M( \pm \delta)$ intersect on the same two diameters at $K( \pm \delta)$ and $M( \pm \gamma)$.

Let

then $K( \pm \alpha)$ becomes

$$
\sigma=\left(\frac{n}{2}+\frac{1}{4}\right) \pi+\vartheta
$$

$(\sin 2 \rho \cos 2 \vartheta-\cos 2 \rho \sin 2 \vartheta \sin 2 \phi)^{2}=\cos ^{2} 2 \phi \cos ^{2} 2 \rho \tan ^{2} 2 \alpha$, and $\mathrm{M}( \pm \beta)$ becomes

$$
(\sin 2 \rho \sin 2 \vartheta+\cos 2 \rho \cos 2 \vartheta \sin 2 \phi)^{2}=\sin ^{2} 2 B \text {. }
$$

From these equations it appears that, in changing the sign of $\phi$, we shall change only the sign and not the magnitude of $\vartheta$; so that if we have drawn part of $K( \pm \alpha)$ or $M( \pm \alpha)$ between the limits $\phi=0$ and $\phi=45^{\circ}$, we can draw the corresponding part of the curve between the limits $\phi=0$ and $\phi=-45^{\circ}$. If $\sigma^{\prime}$ be the value of $\sigma$ corresponding to $-\phi$,

and therefore

$$
\sigma^{\prime}=\left(\frac{n}{2}+\frac{1}{4}\right) \pi-\vartheta
$$

$$
\sigma+\sigma^{\prime}=\left(n+\frac{1}{2}\right) \pi . \quad \text {. . . . . }
$$

Putting $\alpha=0$ and $\beta=0$ in (8) and (9), we get for $\mathrm{K}(0)$,

$\sin 2 \rho \sin 2 \sigma+\cos 2 \rho \cos 2 \sigma \sin 2 \phi=0$; . . and for $\mathrm{M}(0)$,

$$
\sin 2 \rho \cos 2 \sigma-\cos 2 \rho \sin 2 \sigma \sin 2 \phi=0 .
$$

Let $s$ be the value of $\sigma$ where a radius $\phi$ intersects a branch 
of $\mathrm{M}(0)$, and let $s+\xi$ be the value of $\sigma$ where the same radius intersects a branch of $\mathrm{K}( \pm \alpha)$; then by (8) we have

$\{(\sin 2 \rho \sin 2 s+\cos 2 \rho \cos 2 s \sin 2 \phi) \cos 2 \xi$

$+(\sin 2 \rho \cos 2 s-\cos 2 \rho \sin 2 s \sin 2 \phi) \sin 2 \xi) \xi^{2}$

$=\cos ^{2} 2 \rho \cos ^{2} 2 \phi \tan ^{2} 2 \alpha$. . . . . . . .

But by (13) the coefficient of $\sin 2 \xi=0$, and $\xi$ is determined by $\cos 2 \xi$ only, from which it appears that $\xi$ has pairs of values of equal magnitude and opposite sign. Hence, if we have drawn a part of $K( \pm \alpha)$ on one side of a branch of $M(0)$, we can draw the corresponding part on the other side of $M(0)$.

A similar property of $M( \pm \beta)$ with respect to $K(0)$ may be proved in the same manner.

Where $K( \pm \alpha)$ intersects $M(0), \xi$ vanishes and the diameter becomes a tangent. Its position is determined by putting $\beta=0$ in (10). This gives

$$
\cos ^{2} 2 \phi \cos ^{2} 2 \rho=\cos ^{2} 2 \alpha . \quad . \quad . \quad .
$$

The position of the diameter tangents to $M( \pm \beta)$ is determined by putting $a=0$ in the same equation. We get

$$
\cos ^{2} 2 \phi \cos ^{2} 2 \rho=\cos ^{2} 2 \beta \text {. }
$$

Hence the same diameters are tangents to $K( \pm \gamma), M( \pm \gamma)$ at the points where they intersect $M(0)$ and $K(0)$ respectively.

If we put $\sigma \pm 45^{\circ}$ for $\sigma$ in $\mathrm{K}(0)$ we obtain $\mathrm{M}(0)$, and conversely. See equations (12) and (13). . . . . . (17)

If we put $\sigma \pm 90^{\circ}$ for $\sigma$ in $K( \pm \alpha)$ or $M( \pm \beta)$, the equation is unaltered; and by this means from one branch of one of these curves we can obtain all other branches of the curve. (18)

We will now consider the forms of the loci, dealing first with figs. 3 and 4 , in which the incident light is elliptically polarized.

To obtain the isomorphals, we have the equation (7) for $M(\beta)$ and (13) for $M(0)$.

Putting $\beta=\rho$, we obtain the following equation to $M(\rho)$, viz.

$$
\sin \sigma(\sin 2 \rho \sin \sigma+\cos 2 \rho \cos \sigma \sin 2 \phi)=0 ; .
$$

and putting $\beta=-\rho$, we obtain the following equation to $\mathrm{M}(-\rho)$, viz.

$$
\cos \sigma(\sin 2 \rho \cos \sigma-\cos 2 \rho \sin \sigma \sin 2 \phi)=0 . \quad \text {. }
$$

$M(\rho)$ therefore consists of circles for which $\sin \sigma=0$, and ovals intersecting those circles on the diameters $\mathrm{SS}^{\prime}$ and $\mathrm{TT}^{\prime \prime}$; and $\mathrm{M}(-\rho)$ consists of circles for which $\cos \sigma=0$, and ovals intersecting those circles also on $\mathrm{SS}^{\prime}$ and $\mathrm{TT}^{\prime}$. The outer circles in the figures are circles of $\mathrm{M}(\rho)$; and the middle circle is one of the circles of $\mathrm{M}(-\rho)$. 
When $\beta= \pm 45^{\circ}$, the light becomes circularly polarized, and therefore the value of $\alpha$ becomes indefinite; consequently in equation (6) we must have $\cos 2 \phi=0$. Putting this value of $\cos 2 \phi$ in (7), we get the following values of $\phi, \sigma$, and $\beta$ at points where the polarization is circular:-

$$
\left.\begin{array}{rrr}
\phi . & \sigma . & \beta . \\
+45^{\circ}, & n \pi+45^{\circ}+\rho, & -45^{\circ} \\
+45^{\circ}, & n \pi+135^{\circ}+\rho, & +45^{\circ} \\
-45^{\circ}, & n \pi+45^{\circ}-\rho, & +45^{\circ} \\
-45^{\circ}, & n \pi+135^{\circ}-\rho, & -45^{\circ} .
\end{array}\right\} .
$$

These points will be called the "circular points." All the isoclinals pass through all the circular points. The sign of $B$ indicates the direction of rotation of the æther. Where the sign is positive, the direction of rotation has not been altered by the passage of the light through the body; where the sign is negative, the direction of rotation has been reversed.

To draw the isomorphals :-

Mark the circular points by (21). Draw the circles of $\mathrm{M}(\rho)$ and $\mathrm{M}(-\rho)$ by $(19)$ and $(20)$. Draw one branch of $\mathrm{M}(0)$ from $\phi=0$ to $\phi=45^{\circ}$ by (13).

Obtain a branch of $K(0)$ by (17).

Obtain an oval of $\mathrm{M}(\rho)$ between the above limits by (14).

Draw the part of a branch of $M(\beta)$, for example, $M(X V$. in fig. 3 , or $\mathrm{M}(\mathrm{XXX}$.) in fig. 4 , which lies on one side of $\mathrm{M}(0)$, and complete on the other side by $(14)$.

Draw the remaining branches of $\mathrm{M}( \pm \beta)$ between the above limits by (18).

Draw the isomorphals between $\phi=0$ and $\phi=-45^{\circ}$ by (11), and complete the figure by means of the symmetry about $\mathrm{UU}^{\prime}$ and $\mathrm{V} \mathrm{V}^{\prime}$.

Write against the isomorphals the values of $\beta$, taking care to make the sign of $\beta$ the same as that at the nearest circular point.

To draw the isoclinals :-

Equations (6) and (8) are not in a form available for calculation; but by solving (8) as a quadratic in sin $2 \phi$, we obtain the equation to $K( \pm \alpha)$ in the form

$\sin 2 \rho \cos 2 \sigma \sin 2 \sigma \cos ^{2} 2 \alpha+\cos 2 \rho\left(1-\sin ^{2} 2 \sigma \cos ^{2} 2 \alpha\right) \sin 2 \phi$

$$
= \pm \sin 2 \alpha\left(\cos ^{2} 2 \rho-\sin ^{2} 2 \sigma \cos ^{2} 2 a\right)^{\frac{1}{2}}
$$

from which, by putting successive values for $\sigma$, we can obtain corresponding value of $\phi$.

By putting $\alpha=\rho$, we obtain the equation to $\mathrm{K}( \pm \rho)$, viz.

$\sin 2 \rho \cos 2 \sigma-\cos 2 \rho \sin 2 \sigma \sin 2 \phi= \pm \sin 2 \phi$. 
We have already obtained a branch of $K(0)$. Draw from (23) the part of a branch of $K(\rho)$ which lies on one side of $M(0)$ between the limits $\phi=0$ and $\phi=45^{\circ}$, and from (22) obtain a similar part of a branch of $\mathrm{K}( \pm \alpha)$-for example, $K(15)$ in fig. 3 and $K(30)$ in fig. 4 .

Complete these branches by (14). (18).

Complete the other branches between the same limits by

Draw the figure between $\phi=0$ and $\phi=-45^{\circ}$ by (11), and complete the figure by the symmetry about $U^{U^{\prime}}$ and $V V^{\prime}$.

Draw straight lines in the direction $\mathrm{U} \mathrm{U}^{\prime}$ and $\mathrm{VV}^{\prime}$ to give $\mathrm{K}( \pm 45)$, for which the equation is

$$
\sin 2 \phi=0 . \quad \text {. . . . . . }
$$

To number the isoclinals :-

Note in (6) that when $\sigma=n \pi, \tan 2 a=-\tan 2 \phi$, or $\alpha=-\phi$; and when $\sigma=\left(n+\frac{1}{2}\right) \pi, \tan 2 \alpha=\tan 2 \phi$, or $\alpha=+\phi$. Hence the intersections of the isoclinals with the circles of $K(\rho)$ graduate that circle in the negative direction, and their intersections with the circle of $\mathrm{K}(-\rho)$ graduate that circle in the positive direction. Graduate these circles accordingly, taking care to deduct $180^{\circ}$ from the graduation when it exceeds $90^{\circ}$, and $360^{\circ}$ from it when it exceeds $270^{\circ}$, and the readings will give the values of $\alpha$.

The deductions are made to keep the readings low, and for the sake of symmetry.

From the figures 3 and 4 it appears that $\mathrm{M}( \pm \rho)$ divides the figure into regions of two kinds : one kind, which I will call the "segments," contains all the points at which the light is more circularly polarized than the incident light; and the other kind, which I will call the "rings," contains all the points at which the light is more plane-polarized.

In the segments the isomorphals are closed curves surrounding the circular points ; and in the rings the isomorphals are closed curves surrounding the centre of the figure.

It also appears that $K( \pm \rho)$ divides the figure into regions of two kinds-one containing all the points at which both the axes of the ellipse are inclined to the radius by a greater angle than $\rho$, and the other containing all the points at which one of the axes is inclined at a less angle than $\rho$. Both kinds of regions are four-cornered, and have two opposite corners on circular points; but in the first kind both these circular points lie on the same radius, and in the second the circular points lie on different radii. The isoclinals in each region pass from one circular point to the other. 
Comparing fig. 3 with fig. 4, we see that as $\rho$ increases, the segments become smaller, and the isomorphals in the rings become more circular. When $\rho=45^{\circ}$, as in fig. 2 , the segments vanish, and the isomorphals all become circular, the equation to $\mathrm{M}(\beta)$ becoming

$$
\cos 2 \sigma-\sin 2 \beta=0 \text {. }
$$

The circular points are retained in the figure to show its relation to the other figures; but the whole circles through them are loci of circularly polarized light.

The equation to $K( \pm x)$ gives $\tan 2 \alpha=\infty$;

$$
\therefore \alpha= \pm 45^{\circ} \text {. }
$$

The equation to $\mathrm{K}( \pm \rho)$ becomes

$$
\cos 2 \sigma= \pm \sin 2 \phi \text {. }
$$

This curve is retained in the figure to show the continuity with the other figures-although, as the inclination is everywhere $45^{\circ}$ or $-45^{\circ}$, the points on the curve have no special properties. For the same reason the straight lines $K( \pm 45)$ are retained.

Again, comparing figs. 3 and 4 , we see that as $\rho$ diminishes, the segments increase ; and at last, in fig. 5 , where $\rho$ vanishes, the segments fill the whole space and the rings vanish. Each closed curve of $\mathrm{M}(0)$ is forced into a broken line consisting of quadrants of circles joined by pieces of diameters; and as these closed curves now touch at their angles, they form together a complete system of circles and diameters whose equation is, putting $\rho=0$ in (13),

$$
\sin 2 \sigma \sin 2 \phi=0 \text {. }
$$

The isoclinal $K(0)$ also forms a system of circles and diameters; their equation is, from (12),

$$
\cos 2 \sigma \sin 2 \phi=0 \text {. }
$$

All the other isomorphals form closed curves round the circular points; their equation is, from (7),

$$
\sin 2 \sigma \sin 2 \phi+\sin 2 \beta=0 \text {. }
$$

And all the other isoclinals pass from one circular point to another on the same radius; their equation is, from (6),

$$
\cos 2 \sigma \tan 2 \phi+\tan \alpha=0 \text {. }
$$

The diameters of $K(0)$ and $M(0)$ coincide; this is indicated 
in fig. 5 by the thick continuous line having dots on one side of it.

The figure may be drawn in a similar manner to that described for figs. 3 and 4.

The locus of plane-polarized light may be investigated without reference to the condition of the rest of the light, by drawing $\mathrm{M}(0)$ by equation (13), and marking the direction of polarization at successive points on it by equation (15).

In figs. 6, 7, and 8 the points through which short straight lines are drawn are points at which the light is plane-polarized; and the short straight lines through them show the direction of polarization at the points. The dotted lines connecting these points are loci of plane-polarized light. The centres about which small circles are drawn are points at which the light is circularly polarized ; and in fig. 6 the dotted lines connecting these circles are loci of circularly polarized light. The signs within the circles indicate the direction of rotation of the æther; see (21).

In fig. $6, \rho=45^{\circ}$; in fig. $7, \rho=22^{\circ} 30^{\prime}$; in fig. $8, \rho=0$.

If the light be passed through an analyzing Nicol with its plane of polarization inclined at an angle $\rho^{\prime}$ to $\mathrm{SS}^{\prime}$, we can obtain the intensity of the light at any point as follows :-

The vibration along the major axis is $\cos \beta \cos t$, and that along the minor axis is $\sin \beta \sin t$; so that the vibration in the direction of the plane of polarization of the analyzer is

$$
\cos \left(\rho^{\prime}-\alpha\right) \cos \beta \cos t-\sin \left(\rho^{\prime}-\alpha\right) \sin \beta \sin t .
$$

Hence, if $I$ is the intensity of the light after passing the analyzer,

$$
\begin{aligned}
I & =\cos ^{2}\left(\rho^{\prime}-\alpha\right) \cos ^{2} \beta+\sin ^{2}\left(\rho^{\prime}-\alpha\right) \sin ^{2} \beta, \\
2 I & =1+\cos 2\left(\rho^{\prime}-\alpha\right) \cos 2 \beta .
\end{aligned}
$$

The appearance of the light after passing an analyzer might be calculated from this equation, but can be inferred more readily by an inspection of the figures, which show its state before passing.

We notice that two dark spots will be seen on each branch of $\mathrm{M}(0)$, one at each extremity of a diameter, at the points where the vibration is perpendicular to the plane of the analyzer. The spots on the successive branches of $M(0)$ will be alternately on a certain diameter and on the diameter perpendicular to it.

When the incident light is circularly polarized, these spots will move round in circles with unaltered appearance and at a uniform rate as the analyzer is turned uniformly. See figs. 2 and 6. 
When the incident light is elliptically polarized, the spots will move round the curves $\mathrm{M}(0)$; but the rate and appearance will vary (see figs. 3,4 , and 7 ). For on the circles of $\mathrm{M}(\rho)$ the major axis of the ellipse preserves a constant direction in space, since $\phi+a=0$; but on the circles of $M(-\rho)$ the major axis rotates uniformly in. space with an angular velocity double that of the radius, since $\phi-\alpha=0$. Hence in those portions of $\mathrm{M}(0)$ which are near the circles of $\mathrm{M}(\rho)$, the change in the direction of the vibration will be slow; so that in this part the spot will be elongated, and will move more rapidly than the analyzer is rotated: but in the parts of $M(0)$ near the circles of $\mathrm{M}(-\rho)$, the change in the direction of the vibration will be rapid; so that in these parts the spot will be shortened, and will move more slowly than the analyzer is rotated.

When the incident light is plane-polarized (see figs. 5 and 8), the slow-changing parts of $M(0)$ have combined to form the inner and outer circles of the figure and the diameters $\mathrm{S} \mathrm{S}^{\prime}$ and $\mathrm{T} \mathrm{T}^{\prime}$. Along these lines the direction of vibration has no change, but remains constartly the same as that of the incident light; but on the middle circle of the figure, and on corresponding circles, the direction of vibration rotates uniformly with a velocity double that of the radius. Hence on the latter circles there will be spots moving uniformly round with a velocity double that of the analyzer; but on the other parts of the figure there will be no spots. However, when the spots on the latter circles reach the diameters, then the former circles and the diameters will become black.

If the light is not monochromatic, these appearances will not be so distinctly seen, as the absence of one colour will not occur exactly in the same place as the absence of another, since the position of the isomorphal and isoclinal lines depends upon the value of $\sigma$, and this will differ for different colours. But the position of diameters which give planepolarized light in figs. 5 and 8 , is not dependent on the value of $\sigma$; and hence with any light this cross will always appear uncoloured, being black when the upper and lower Nicols are crossed, and in full light when they are parallel.

If $\sigma$ is constant, the isomorphals and isoclinals become straight lines from the centre, and the state of the polarization may be conveniently represented by taking a series of points in a circle round the centre, and drawing about each point the ellipse of polarization at that point. The ellipse will show the polarization along the radius on which it lies.

Plil. Mag. S. 5. Vol. 7. No. 40. Jan. 1879. 
This is done in figures 9 to 13 , in which $\sigma$ is about $15^{\circ}$. In fig. $9, \rho=45^{\circ}$; in fig. $10, \rho$ lies between $45^{\circ}$ and $\sigma$; in fig. 11 , $\rho=\sigma$; in fig. 12, $\rho$ lies between $\sigma$ and zero ; and in fig. 13, $\rho$ is zero.

Suppose now the light to be passed through an analyzer placed with its plane of polarization in the direction T $\mathrm{T}^{\mathrm{V}}$. When $\rho=45^{\circ}$, the quadrants about $\mathrm{U}^{\prime}$ will be dark, and those about $V^{\prime} V^{\prime}$ will be light. They will gradually shade into one another, there being no black or full light. As $\rho$ diminishes, $\mathrm{UU}^{\prime}$ becomes darker until, when $\rho=\sigma$, $\mathrm{UU}^{\prime}$ is black (see fig. 11). As $\rho$ further diminishes, the black bar opens out into a dark oblique cross, neither bar of which is black; and when $\rho$ becomes zero, this cross becomes rectangular and black. As $\rho$ passes on to $-\sigma$, the cross becomes oblique and not black, and closes up into a black bar along $V V^{\prime}$; and when $\rho$ becomes $-45^{\circ}$, the quadrants about $V V^{\prime}$ are dark, and those about $\mathrm{U}^{\prime} \mathrm{U}^{\prime}$ light. When we have the oblique cross, we can by a suitable turn of the analyzer make either arm of the cross black. (See fig. 12.)

If the analyzer is placed with its plane of polarization in the direction $\mathrm{S} \mathrm{S}^{\prime}$, we get the same set of appearances, except that we get light for dark and dark for light; and in the case of the single bar and rectangular cross, we get full light instead of black.

The appearances presented when $\sigma$ is variable may be well seen in cylindrical disks of unannealed glass. I do not know of any bodies which show very clearly the appearances presented when $\sigma$ is constant. Crystals of salicene show the black cross remarkably well, and give indications of the single black bar; but in this substance $\sigma$, though constant along each radius, varies in passing from one radius to another, and this completely hides the phenomena of the oblique cross. However, in grains of tous-les-mois starch, phenomena closely analogous to those above described as presented when $\sigma$ is constant may be easily observed under a moderately high power-the only difference in the phenomena being that, in consequence of the grain of starch being generally an unsymmetrical body, the lines are distorted, the black cross, for instance, being neither rectangular nor rectilinear. See "The Optical Properties of Starch," Phil. Mag. for August 1876. 
Phil Mag. S. 5.Vol. 7. Pl II

Fig: 2

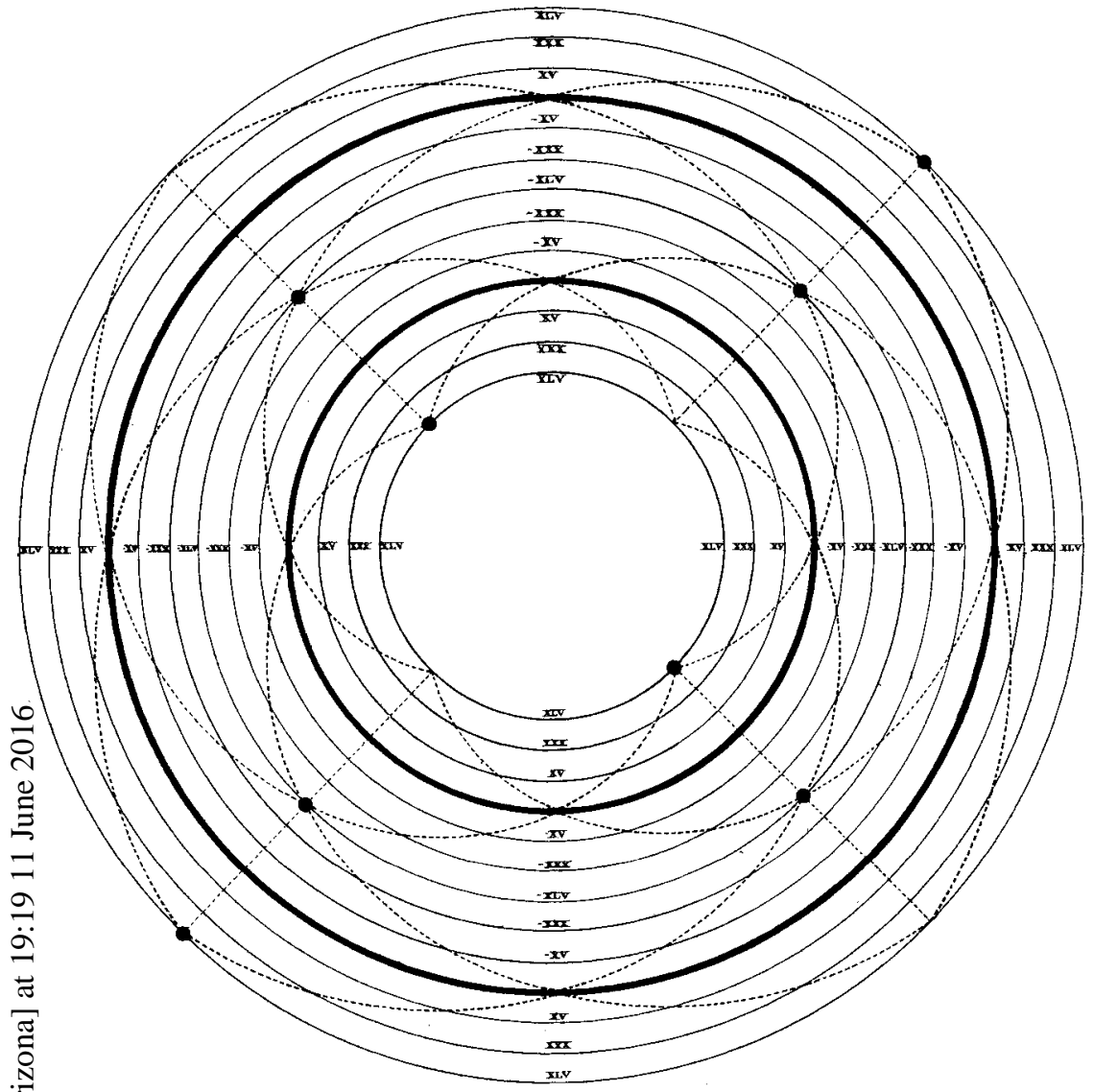

Fig: 3 .

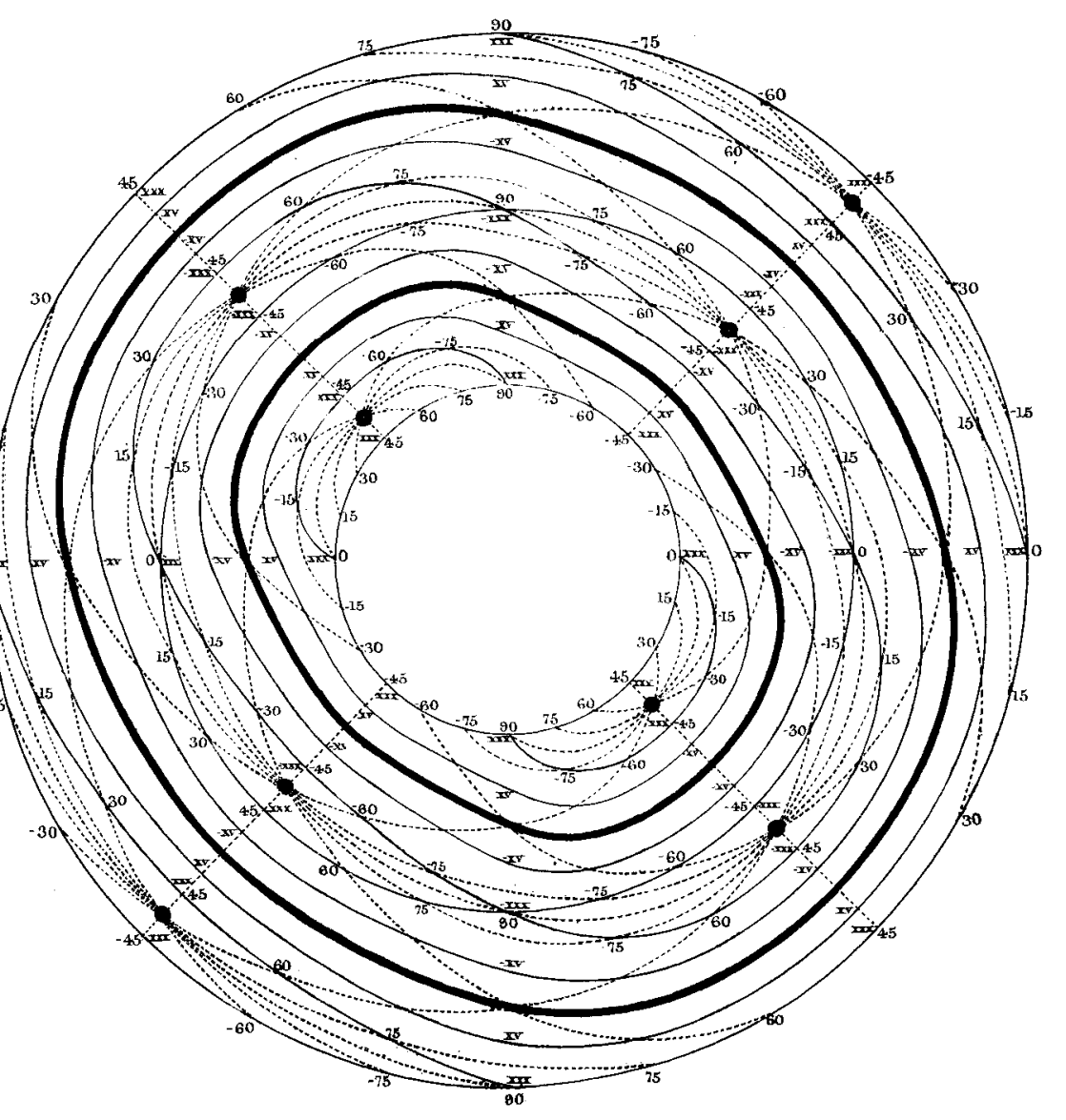


Phil.Mag. S.5.Vo1.7 .P1.III

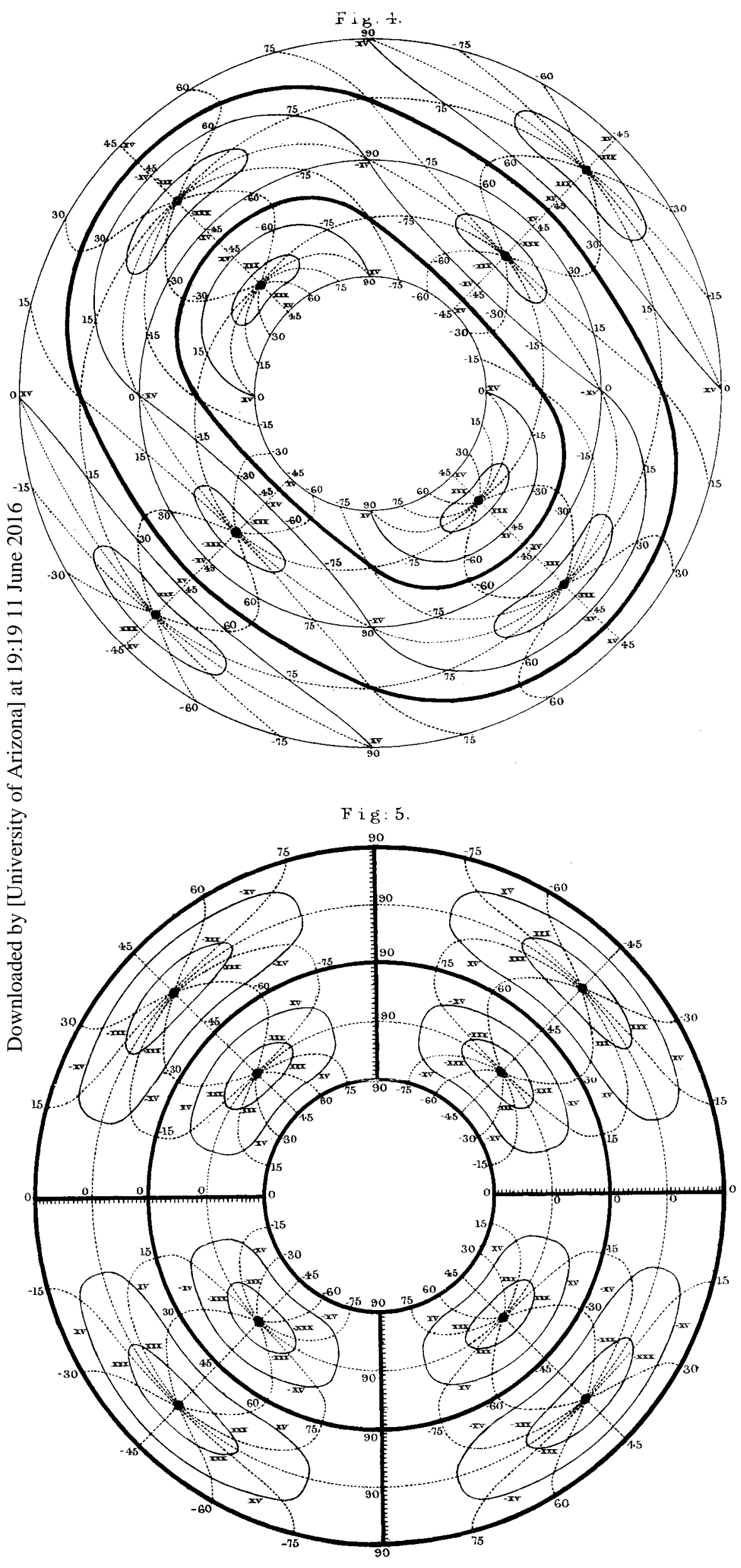


